\title{
Pekar bipolaron and the virial theorem (arbitrary coupling)
}

\author{
N.I. Kashirina \\ V. Lashkaryov Institute of Semiconductor Physics, NAS of Ukraine; \\ 41, prospect Nauky, 03028 Kyiv, Ukraine \\ E-mail:n_kashirina@mail.ru
}

\begin{abstract}
The work is devoted to issues related with implementation of the virial theorem for one-center bipolaron. The virial theorem expressions have been obtained for an electron system with Coulomb interactions in the phonon field. It is shown that for the bipolaron functional (one-center configuration) virial theorem holds for arbitrary electron-phonon coupling. As a specific example of the virial theorem for one-center bipolaron configuration, the author adduces numerical calculations of the energy of the ground state and the various contributions (kinetic energy, electron-phonon interaction, electron energy, phonon energy) into the energy of bipolaron, performed within the framework of Buimistrov-Pekar method. It is shown that the virial theorem is fulfilled with high accuracy for the two-electron systems with Coulomb interactions for an arbitrary value electron-phonon coupling. The necessary condition for formation of a bipolaron stable state is accounting electron correlations associated with the direct dependence of the trial electron wave function of the system from the interelectron distance.
\end{abstract}

Keywords: bipolaron, Fröhlich Hamiltonian, electron-phonon interaction, electron correlations, superconductivity, high-temperature superconductivity.

Manuscript received 03.06.14; revised version received 00.00.14; accepted for publication 00.00.14; published online 00.00.14.

\section{Introduction}

The question of whether a bound state of two polarons is possible is closely related to finding a potential of pairwise interaction between two polarons, which is a function of the distance between them. For large-radius bipolaron, the region of existence of a bound state is limited, on the one hand, by the Fröhlich constant of electron-phonon interaction, to rather a large $\alpha_{c}=6.8$ [1], below which bound bipolaron state does not exist. In the paper [1], calculations were carried out using the Feynman method of integration over trajectories, which yields translation invariant solutions of polaron and bipolaron problems. The methods that use a direct variation of the bipolaron wave function give a slightly higher value of the electron-phonon interaction parameter: $\alpha_{c}=7.3[2,3]$, and $\alpha_{c}=6.9$ [4]. These values were obtained in finding the bipolaron coupling energy
$\Delta E_{B p}=2 E_{P}-E_{B p}$ (where $E_{P}$ and $E_{B p}$ are the energies of the polaron and bipolaron ground states, respectively) with respect to the lower boundary of the polaron energy for $\eta=\varepsilon_{\infty} / \varepsilon_{0} \rightarrow 0$.

The idea to explain the high-temperature superconductivity by Bose condensation of singlet electron pairs localized in spherically symmetric cavities formed in metal ammonia solutions, belongs to Ogg [5-7]. Later on S.I. Pekar [8] introduced a model of one-center bipolaron as a bound state of two electrons in an ionic crystal. In that model, the two-center wave function was constructed by analogy with helium atom

$\Psi\left(\mathbf{r}_{1}, \mathbf{r}_{2}\right)=N\left(1+\alpha r_{1}\right)\left(1+\alpha r_{2}\right) \times$
$\times\left(1+\beta r_{12}\right) \exp \left(-\alpha r_{1}\right) \exp \left(-\alpha r_{2}\right)$,

where $N$ is a normalizing multiplier; $\mathbf{r}_{1}, \mathbf{r}_{2}$ are coordinates of first and second electrons, respectively, $r_{12}=\left|\mathbf{r}_{1}-\mathbf{r}_{2}\right|$ is the distance between first and second 
electrons, $\quad r_{1}=\left|\mathbf{r}_{1}\right|, \quad r_{2}=\left|\mathbf{r}_{2}\right| ; \quad \alpha, \quad \beta \quad$ are variational parameters.

For the first time, calculations of the model were carried out by S.I. Pekar's PhD fellow, O.F. Tomasevich, for the case of bound bipolaron or $F^{\prime}$ center [9].

The mistakes made in calculating the normalizing multiplier of the wave function chosen in the form of (1) and in finding the phonon contribution into the bipolaron functional led to underestimation of the $F^{\prime}$-center binding energy and erroneous conclusion about an insignificant role of interelectron correlations associated with the direct dependence of the wave function on the distance between electrons. Later on, referring to [9], S. I. Pekar [8] made a conclusion that stable bipolaron cannot be formed in the framework of the one-center model. Function (1) was also used by A.S. Davydov [10] to calculate the bipolaron energy in metal-ammonia solution. The erroneous conclusion about the instability of one-center bipolaron was made in that work, too. The failed attempts to find a bound bipolaron state in the framework of one-center configuration gave rise to a model of two-center bipolaron [11, 12]. Earlier polaron effects in ionic crystals were studied for the case of two centers system $\left(F_{2}\right.$-center $)$ in [13]. That work provided the basis for the bipolaron model suggested in [11] where the electron wave function was chosen by complete analogy with a hydrogen molecule

$\Psi\left(\mathbf{r}_{1}, \mathbf{r}_{2}\right)=\Phi\left(\mathbf{r}_{1}, \mathbf{r}_{2}\right)+\Phi\left(\mathbf{r}_{2}, \mathbf{r}_{1}\right)$.

In the pioneering paper aimed at calculation of the bipolaron energy, the wave function $\Phi\left(\mathbf{r}_{1}, \mathbf{r}_{2}\right)$ was chosen as a product of hydrogen-like wave functions centered at different points coinciding with the centers of polarization wells of two polarons [11]. In the next paper [12] that dealt with two-center bipolaron, the polaron functions were chosen in a more general form, and the wave function $\Phi\left(\mathbf{r}_{1}, \mathbf{r}_{2}\right)$ was a product of Pekar's polaron functions $\quad\left(1+\alpha r_{a 1}\right) \exp \left(-\alpha r_{a 1}\right)\left(1+\alpha r_{b 2}\right) \exp \left(-\alpha r_{b 2}\right)$ where the traditional notation is used for the two-center coordinate system: $\mathbf{r}_{a 1}\left(\mathbf{r}_{a 2}\right)$ is the radius-vector of first (second) electron reckoned from the center $a, \mathbf{r}_{b 1}\left(\mathbf{r}_{b 2}\right)$ is the same for the center $b$.

An alternative method of finding the bipolaron energy was used in [14], the authors of which suggested a model Hamiltonian for calculation of the two-center bipolaron energy by integration over trajectories. There, a variational parameter was introduced, which could be treated as a distance between the centers of polarization wells for two polarons. The model was studied qualitatively; numerical calculations were not carried out.

For more than thirty years that passed since the time when the pioneering papers dealt with calculation of the strong-coupling bipolaron energy $[11,12]$ were published till the time when the paper by Suprun and Moyzhes [15] came out where the numerical mistakes made in [9] and [10] were corrected, the object of investigations was just two-center bipolaron, notwithstanding the small value of the coupling energy.
After the paper [15] had come out, and its results had been reproduced by various research teams with the use of various methods and also in view of the fact that the one-center bipolaron configuration is energetically more advantageous than the two-center one, investigations of the latter practically ceased. The author of [16] erroneously (as we will show below) states that the virial theorem is violated in the case of one-center bipolaron and holds true only in the case of a two-center configuration. For this reason, the author [16] erroneously claims that the one-center bipolaron configuration corresponds to a formal solution that has no physical meaning. The proof that the virial theorem is fulfilled in the strong coupling limit for the one-center bipolaron configuration was carried out in [17]. In this paper, we performed a study of the virial theorem for the one-center bipolaron configuration in the case of arbitrary electron-phonon coupling.

\section{Basic relations}

In this paper, we deal only with the theory of largeradius continuum bipolaron. The electron-phonon interaction will be described by Fröhlich Hamiltonian. For two electrons in a phonon field, the Hamiltonian has the form:

$$
\begin{aligned}
& H_{B p}=-\frac{\hbar^{2}}{2 m^{*}}\left(\Delta_{1}+\Delta_{2}\right)+\frac{e^{2}}{\varepsilon_{\infty}\left|\mathbf{r}_{1}-\mathbf{r}_{2}\right|}+ \\
& \hbar \omega \sum_{\mathbf{k}} a_{-\mathbf{k}}^{+} a_{\mathbf{k}}+\sum_{\mathbf{k}} V_{\mathbf{k}}\left(a_{\mathbf{k}}-a_{\mathbf{k}}^{+}\right)\left(\exp \left(i \mathbf{k} \mathbf{r}_{1}\right)+\exp \left(i \mathbf{k} \mathbf{r}_{2}\right)\right),
\end{aligned}
$$

where

$\Delta_{i}=\frac{\partial}{\partial x_{i}^{2}}+\frac{\partial}{\partial y_{i}^{2}}+\frac{\partial}{\partial z_{i}^{2}}, i=1,2 ; \quad \mathbf{r}_{i}=\left\{x_{i}, y_{i}, z_{i}\right\}$,

$V_{k}=-i \frac{e}{k} \sqrt{\frac{2 \pi \hbar \omega}{V \widetilde{\varepsilon}}}, \frac{1}{\widetilde{\varepsilon}}=\frac{1}{\varepsilon_{\infty}}-\frac{1}{\varepsilon_{0}}$,

$\omega$ is the frequency of optical phonons, $\mathbf{k}$ - wave vector of phonons, $a_{k}^{+}$and $a_{k}$ are operators of birth and annihilation of phonons with the wave vector $\mathbf{k}, m^{*}$ and $e$ - electron's effective mass and charge respectively, $\varepsilon_{0}$ and $\varepsilon_{\infty}-$ static and high-frequency dielectric permittivities, $V$ is the crystal volume.

Though the analogy with a hydrogen molecule provided the basis for the pioneering papers aimed at calculation of strong-coupling bipolaron [11, 12], the bipolaron Hamiltonian has an essential peculiarity that distinguishes it from the Hamiltonian of a hydrogen molecule. Namely, in Hamiltonian (3) the parameter analogous to the distance between two protons in the molecule is lacking. In molecular physics, of equally frequent use are the dependences of the hydrogen molecule energy on the distance between protons of two types: with and without regard for repulsion between protons (for example Figs 3.3 and 3.4 in the ref. [18], correspondingly). The latter dependences are especially suitable for finding the energy of a system consisting of 
two hydrogen atoms on the small distances between protons by using variational calculations and for getting a limit transition corresponding to joining of two hydrogen atoms into helium atom. In this case, the distance dependence of the hydrogen molecule energy has the only minimum corresponding to configuration of helium atom at the point $R=0$. In other words, the dependence of the wave function of the hydrogen molecule system on the distance between protons does not lead to arising of an energy minimum at the point $R=R_{m} \neq 0$. The reason is just that the Hamiltonian of a hydrogen molecule contains a term representing repulsion between protons.

Since Hamiltonian of two electrons in a phonon field (3) does not depend on the distance between the centers of polarization wells for two polarons, one would assume that potential dependences of the total energy of the system on the distance between polarons should correspond to potential dependences of the hydrogen molecule energy on the distance between protons without regard for repulsion between protons. Thus, the ground state of the two-electron system should correspond to a spherically-symmetric state.

\section{The problem of taking account of electronic correlations and spatial configuration of a bipolaron}

In the continuum approximation in the strong coupling limit polaron and bipolaron functionals have the form $[8,15]$

$$
\begin{aligned}
& J_{P}=\frac{T_{1}}{N_{P}}-\frac{1}{2 N_{P}^{2}} C \int \frac{\Psi_{1}^{2} \Psi_{2}^{2}}{r_{12}} d \tau_{12}, \\
& J_{B p}=\frac{T_{12}}{N}+\frac{e_{0}^{2} J_{C}}{\varepsilon_{\infty} N}-\frac{2 e_{0}^{2} C}{N^{2}} \int \frac{\Psi_{12}^{2} \Psi_{34}^{2}}{r_{13}} d \tau_{12} d \tau_{34}, \\
& T_{1}=-\hbar^{2} / 2 m^{*} \int \Psi_{1} \Delta_{1} \Psi_{1} d \tau_{1}, \\
& T_{12}=-\hbar^{2} / 2 m^{*} \int \Psi_{12}\left(\Delta_{1}+\Delta_{2}\right) \Psi_{12} d \tau_{12}, \\
& J_{C}=\int \frac{\Psi_{12}^{2}}{r_{12}} d \tau_{12}, \Psi_{i} \equiv \Psi_{i}\left(r_{i}\right), \Psi_{i j} \equiv \Psi\left(r_{i}, r_{j}\right),
\end{aligned}
$$

where $N_{P}, N$ are normalizing multipliers of the polaron and bipolaron wave functions, respectively; $C=\left(\varepsilon_{\infty}^{-1}-\varepsilon_{0}^{-1}\right) ; r_{12}$ is the distance between electrons.

In what follows, we will use effective Hartree $H a^{*}=\hbar^{2} / a_{0}{ }^{2} m^{*}$ as a unit of energy and effective Bohr radius $a_{0}=\hbar^{2} \widetilde{\varepsilon} / m^{*} e^{2}, \quad \widetilde{\mathcal{\varepsilon}}^{-1}=\varepsilon_{\infty}^{-1}-\varepsilon_{0}^{-1}$ as a unit of length.

The condition necessary to form a bound onecenter bipolaron state is the availability of interelectron correlations, which can be taken into account, for example, by the direct dependence of the wave function on the distance between electrons.

The reason is that in choosing the electron wave function in the multiplicative form, even in the limit of the strongest coupling when $\eta \rightarrow 0$, the bipolaron functional identically falls into a sum of two polaron functionals.

Interelectron correlations associated with the direct dependence of the electron wave function on the distance between these electrons were taken into account in many papers. As an example, we can write

$\Psi_{12} \sim\left(1+C r_{12}\right)\left(1+b\left(r_{1}+r_{2}\right)\right) \exp \left(-\delta\left(r_{1}+r_{2}\right)\right) \cosh \left(\xi r_{12}\right)[19]$, $\Psi_{12}=\sum_{i}\left(1+P_{12}\right) C_{i} \exp \left(-a_{1 i} r_{1}-2 a_{2 i} r_{12}-a_{3 i} r_{2}\right)[2,3]$,

$\Psi_{12} \sim\left(1-k \exp \left(-\xi\left(r_{1}-r_{2}\right)^{2}\right) \exp \left(-\delta\left(r_{1}^{2}+r_{2}^{2}\right)\right)[20]\right.$, $\Psi_{12} \sim\left(1+k r_{12}^{2}\right) \exp \left(-\delta\left(r_{1}^{2}+r_{2}^{2}\right)\right)[21]$,

$\tilde{N}, b, \delta, \xi, C_{i}, a_{1 i}, a_{2 i}, a_{3 i}, k$ are variational parameters, $P_{12}$ is the electron coordinates permutation operator.

To find the energy of one-center bipolaron, use was made of different two-electron functions. A correct functional of Pekar's bipolaron in the limit of strong electron-phonon interaction is given in [17].

For the case of strong coupling, the bipolaron energy expressed as a function of a distance between polarons was studied in [22] where the dependences of the bipolaron energy on the distance between the centers of polarization wells for two polarons are given for various values of the parameter $\eta$. In the paper [23], similar dependences are presented for various constants of electron-phonon interaction. Calculations were carried out by Buimistrov-Pekar method [24].

\section{Virial theorem and interaction between polarons}

The conditions under which the virial theorem holds true for the polaron functional were considered in [25] in the general case and in [17] as applied to the strong coupling bipolaron functional.

In the strong couplings limit, the energies of the polaron $E_{P}$ and bipolaron $E_{B p}$ ground states are determined as the lower boundary (absolute minimum) of functionals (4) and (5), $E_{P}=\min J_{P}\left[\Psi_{1}\right]$, $E_{B p}=\min J_{B p}\left[\Psi_{12}\right]$. The only additional condition is that the probe wave function should be normalized ([26], p. 156).

Let us replace the probe function $\Psi(\vec{r})$ (where $\vec{r}$ stands for $\vec{r}_{1}, \vec{r}_{2}, \vec{r}_{N}, N$ is the number of electrons) by the function $\psi=\lambda^{3 N / 2} \Psi(\lambda \vec{r})$. The scaling transformation coefficient $\lambda$ is considered as a variational parameter. If the function $\Psi(\vec{r})$ leads to an extremum of the studied functional, then for $\lambda=1$ turns into $\psi(\lambda=1, \vec{r})=\Psi(\vec{r})$, therefore $J_{B p}[\psi]$ should have an extremum for $\lambda=1$.

Having varied functional $J_{B p}[\psi(\lambda \vec{r})]$ with respect to parameter $\lambda$, we get

$$
\frac{d J_{B p}}{d \lambda}=0
$$

\section{(C) 2014, V. Lashkaryov Institute of Semiconductor Physics, National Academy of Sciences of Ukraine}


The formula (8) is a mathematical expression of the virial theorem associated with scaling transformation of the studied system coordinates. It is fulfilled for any extremum of the initial functional including any subsidiary minimum.

In the strong coupling limit, the terms standing for kinetic and potential energies in functionals (4) and (5) possess the property of homogeneity. In this relation, application of scaling transformation enables us to perform variation with respect to the parameter $\lambda$ in an analytical form and to get simple expressions for the ground state energy for which the virial theorem in the simplest form holds true at the extremum points. Namely, the kinetic energy of the system is equal to the negative total energy. For normalizing functions of polaron and bipolaron, the scaled multiplier and the energy of the self-consistent ground state are determined by the expressions:

$$
\begin{aligned}
& \lambda_{P}=-\frac{V_{P}}{2 T_{P}}, \quad \lambda_{B p}=-\frac{V_{B p}}{2 T_{B p}}, \\
& E_{P}=-\min \left\{\frac{V_{P}^{2}}{4 T_{P}}\right\}, E_{B p}=-\min \left\{\frac{V_{B p}^{2}}{4 T_{B p}}\right\},
\end{aligned}
$$

where $E_{P(B p)}, T_{P(B p)}$ are the mean values of the total and kinetic energies of polaron and bipolaron, respectively. In the general case, when some additional Coulomb interactions of bound polarons and bipolarons take place in the system (such as $F$ and $F^{\prime}$-centers in polar crystals), the mean value of the potential energy $V_{P(B p)}$ includes the energy of the phonon field, the energy of electronphonon field and also all Coulomb interactions in the system.

Naturally, the condition (8) should be fulfilled for any constant of the electron-phonon interaction. However, we cannot vary the functionals of intermediate coupling polaron and bipolaron with respect to parameter $\lambda$ and get the scaling transformation multiplier in an analytical form. Nevertheless, at the points corresponding to the minimum of polaron and bipolaron functionals the virial theorem should hold true and can be checked numerically.

As applied to polaron, the virial theorem was studied in S.I. Pekar's paper [8] and obtained the name of 1:2:3:4 theorem. The theorem states that in the strong coupling limit the following relation:

$T_{P}=-F_{P}, E_{P h}=-2 F_{P}$,

$-E_{e l}=-3 F_{P},-E_{\text {int }}=-4 F_{P}$,

is valid, where

$F_{P}=T_{P}+\frac{1}{2} E_{\mathrm{int}}, E_{e l}=T_{P}+E_{\mathrm{int}}$.

The ground state energy $E_{P}$ and the quantities $T_{P}$, $E_{P h}, E_{\text {int }}$ corresponding to the mean values of the kinetic energy, energy of the phonon field and energy of electron-phonon interaction, respectively, are given by the expressions:

$$
\begin{aligned}
& E_{P}=\left\langle\Psi_{P} H_{P} \Psi_{P}\right\rangle, T_{P}=\left\langle\Psi_{P}\left|-\frac{\hbar^{2}}{2 m^{*}} \Delta\right| \Psi_{P}\right\rangle, \\
& E_{P h}=\left\langle\Psi_{P}\left|\sum_{k} \hbar \omega_{k} a_{k}^{+} a_{k}\right| \Psi_{P}\right\rangle, \\
& E_{\text {int }}=\left\langle\Psi_{P}\left|\sum_{k}\left[V_{k} a_{k} \exp (i \vec{k} \vec{r})+V_{k}^{*} a_{k}^{+} \exp (-i \vec{k} \vec{r})\right]\right| \Psi_{P}\right\rangle
\end{aligned}
$$

Since in the strong coupling limit at the minima points, the total polaron energy $E_{P}$ is equal to the negative kinetic energy, the quantity $F_{P}$ determined by expression (12) coincides with the total energy of the self-consistent state, which can be treated as the energy of polaron thermoionization.

The authors of [25] cite evidence that for polaron, the relation 1:3:4 of the theorem remains valid for the whole range of variation of the electron-phonon coupling constant, while the relation 1:2 as well as the equality $F_{P}=E_{P}$ is valid only in the strong coupling limit. Relation 1:2 of the theorem results from the fact that the ground state should be proportional to $\alpha^{2}$ and corresponds to the strong coupling limit considered by S.I. Pekar [8]. As shown in [27], if the polaron energy can be presented as an expansion in terms of parameter $1 / \alpha^{2}$, the theorem 1:2:3:4 remains valid for the terms proportional to $\alpha^{2}$.

Expressions (11) can be obtained if we apply the Feynman theorem to the polaron functional [28]. Recall that according to the theorem, when the value of $E_{0}=\left\langle\Phi_{0} H \Phi_{0}\right\rangle$ depends on the parameter $\beta$, then the derivative with respect to the parameter is given by

$$
\frac{d E_{0}}{d \beta}=\left\langle\Phi_{0} \frac{d H}{d \beta} \mid \Phi_{0}\right\rangle .
$$

The parameter can be the electron charge, effective mass, phonon energy, etc. The development of expressions (11) by using the Feynman theorem (14) is given in the paper [25]. There, the quantities $1 / \mathrm{m}^{*}$ and electron charge are successively considered as parameters.

This technique can be applied to a system containing Coulomb interactions. Performing some simple transformations, we can show that for oneelectron and two-electron (in the general case manyelectron) systems in a phonon field, the theorem 1:3:4 holds true for any value of the electron-phonon interaction. In the case under consideration, it has a more general form

$$
T_{P}=-\widetilde{F},-\widetilde{E}_{e l}=-3 \widetilde{F},-E_{\mathrm{int}}=-4 \widetilde{F},
$$

where use is made of a new notation generalizing Eq. (12) to the case of systems with Coulomb interactions 
$\widetilde{F}=T+\frac{1}{2} E_{\text {int }}+E_{\mathrm{C}}, \quad \widetilde{E}_{e l}=T+E_{\text {int }}+2 E_{\mathrm{C}}$,

where $T$ is a kinetic energy, $E_{\text {int }}$ is the energy of the electron-phonon interaction, $E_{\mathrm{C}}=\left\langle\Phi_{0} H_{\mathrm{C}} \mid \Phi_{0}\right\rangle$ contains all Coulomb interactions in the system.

For the bipolaron functional

$E_{B p}=\left\langle\Phi_{12}\left|H_{B p}\right| \Phi_{12}\right\rangle$,

$T_{B p}=\left\langle\Phi_{12}\left|-\frac{\hbar^{2}}{2 m^{*}}\left(\Delta_{1}+\Delta_{2}\right)\right| \Phi_{12}\right\rangle$,

$E_{P h}=\left\langle\Phi_{12}\left|\sum_{\mathbf{k}} \hbar \omega_{\mathbf{k}} a_{\mathbf{k}}^{+} a_{\mathbf{k}}\right| \Phi_{12}\right\rangle$,

$E_{\mathrm{C}}=\left\langle\Phi_{12}\left|\frac{e^{2}}{\varepsilon_{\infty} r_{12}}\right| \Phi_{12}\right\rangle$,

$E_{\text {int }}=\left\langle\Phi_{12}\right| \sum_{\mathbf{k}, i=1,2}\left[V_{\mathbf{k}} a_{\mathbf{k}} \exp \left(i \mathbf{k r}_{i}\right)+\right.$

$\left.+V_{\mathbf{k}}^{*} a_{\mathbf{k}}^{+} \exp \left(-i \mathbf{k r}_{i}\right)\right]\left|\Phi_{12}\right\rangle$,

where $\Phi_{12}$ is the wave function of the system with Hamiltonian (3).

The problem of calculation of the quantities involved in the expressions (15) deserves special consideration.

By way of example, let us consider calculation of the energy of polaron and bipolaron states in the framework of Buimistrov-Pekar method [24]. The bipolaron probe function $\Phi_{12}$ is chosen in the form:

$\left.\Phi_{12}=\Psi_{12} \exp \left(-S_{1}\right) \exp \left(-S_{2}\right) 0\right\rangle, S_{1}=\sum_{k} F_{k}\left(a_{k}^{+}-a_{k}\right)$

$S_{2}=\sum_{k, i=1,2}\left\{g_{k} \exp \left(-i \mathbf{k} \mathbf{r}_{i}\right) a_{k}^{+}-H . C.\right\}$,

where $\Psi_{12}$ is the electron wave function, $|0\rangle$ is the wave function of the phonon vacuum state, $F_{k}$ and $g_{k}$ are variational functions.

When calculating the mean values of the quantities involved in expressions (17), we can conveniently employ useful expressions relating the operators of phonon field and kinetic energy before and after canonical transformation:

$U^{-1} a_{\mathbf{k}}^{+} U=a_{\mathbf{k}}^{+}-F_{\mathbf{k}}-\left[\exp \left(-i \mathbf{k} \cdot \mathbf{r}_{1}\right)+\exp \left(-i \mathbf{k} \cdot \mathbf{r}_{2}\right)\right] g_{\mathbf{k}}$,

$U^{-1} \frac{1}{i} \nabla_{1,2} U=\frac{1}{i} \nabla_{1,2}+$

$+\sum_{\mathbf{k}} \mathbf{k} g_{\mathbf{k}}\left[\exp \left(-i \mathbf{k} \cdot \mathbf{r}_{1,2}\right) a_{\mathbf{k}}^{+}+\exp \left(-i \mathbf{k} \cdot \mathbf{r}_{1,2}\right) a_{\mathbf{k}}\right]$,

where $U=\exp (-S)=\exp \left(-S_{1}\right) \exp \left(-S_{2}\right)$.

That is to say, in checking whether the virial theorem determined by (15), (16) holds true, we should first perform a canonical transformation corresponding to a particular method and get the expressions corresponding to the new values of the operators for kinetic energy and electron-phonon interaction, and only after that carry out averaging over the electron wave functions.

\section{Numerical illustration of the virial theorem for bipolaron (arbitrary electron-phonon interaction)}

To verify the virial theorem validity for bipolaron, let us introduce the following notation:

$\mathbf{H}=\mathbf{H}_{\mathrm{kin}}+\mathbf{H}_{\mathrm{C}}+\mathbf{H}_{\mathrm{int}}+\mathbf{H}_{P h}$,

$\mathbf{H}_{\text {kin }}=\frac{\mathbf{p}_{1}^{2}}{2 m}+\frac{\mathbf{p}_{2}^{2}}{2 m}, \mathbf{H}_{\mathrm{C}}=\frac{e^{2}}{\varepsilon_{\infty} r_{12}}$,

$\mathbf{H}_{\text {int }}=\sum_{k, i=1,2}\left(V_{\mathbf{k}} \mathbf{a}_{\mathbf{k}} \mathrm{e}^{i \mathbf{k} \mathbf{r}_{i}}+V_{\mathbf{k}}^{*} \mathbf{a}_{\mathbf{k}}^{+} \mathrm{e}^{i \mathbf{k} \mathbf{r}_{i}}\right), \mathbf{H}_{P h}=\sum_{\mathbf{k}} \hbar \omega_{\mathbf{k}} \mathbf{a}_{\mathbf{k}} \mathbf{a}_{\mathbf{k}}^{+}$.

The bipolaron ground state energy is given by:

$E^{0}=\left\langle\Phi_{0} \mid \mathbf{H} \Phi_{0}\right\rangle$.

We also define the following average values as

$E_{\mathrm{C}}=\left\langle\Phi_{0}\left|\mathbf{H}_{\mathrm{C}}\right| \Phi_{0}\right\rangle$,

$E_{\text {int }}=\left\langle\Phi_{0}\left|\mathbf{H}_{\text {int }}\right| \Phi_{0}\right\rangle$,

$\widetilde{E}_{\text {int }}=\left\langle\Phi_{0}\left|\mathbf{H}_{\text {int }}\right| \Phi_{0}\right\rangle+2 E_{\mathrm{C}}$,

$E_{P h}=\left\langle\Phi_{0}\left|\mathbf{H}_{P h}\right| \Phi_{0}\right\rangle$,

$F=E_{\text {kin }}+\frac{1}{2} \widetilde{E}_{\text {int }}$,

$E_{e l}=E_{\mathrm{kin}}+\widetilde{E}_{\mathrm{int}}$,

$E_{P h}^{\mathrm{C}}=E_{P h}-E_{\mathrm{C}}$.

Using the above designations, the virial theorem for bipolaron can be represented as:

$E_{\text {kin }}=-F, E_{P h}^{\mathrm{C}}=-2 F$,

$-E_{e l}=-3 F,-\widetilde{E}_{\mathrm{int}}=-4 F$.

In the strong coupling limit, the theorem holds $\widetilde{1}, \widetilde{2}, \widetilde{3}, \widetilde{4}$ and in the general case for an arbitrary communication theorem $\tilde{1}, \tilde{3}, \tilde{4}$. Signs waves above figures indicate that the system has a Coulomb interaction. We introduced similar notations for convenience to distinguish from bipolaron and polaron.

In the framework of Buimistrov-Pekar method, the energy of the self-consistent state polaron (bipolaron) can be separated into terms that correspond to addition of the strong coupling and addition of an intermediate coupling:

$$
\begin{aligned}
& E_{p(b)}+\delta E_{p(b)}=T_{p(b)}+\delta T_{p(b)}+E_{\mathrm{C}}^{p(b)}+ \\
& +E_{\mathrm{int}}^{p(b)}+\delta E_{\mathrm{int}}^{p(b)}+E_{P h}^{p(b)}+\delta E_{P h}^{p(b)}
\end{aligned}
$$




$$
\begin{aligned}
& \delta E_{p(b)}=\delta T_{p(b)}+\delta E_{\mathrm{int}}^{p(b)}+E_{P h}^{p(b)}= \\
& -\sum_{k} V_{k}^{2} \frac{\left.\left\langle f_{p(b) k} L_{p(b) k}^{*}\right\rangle-\left\langle f_{p(b) k}\right\rangle L_{p(b) k}^{*}\right\rangle^{2}}{t_{k}^{p(b)}+\omega\left(f_{p(b) k} f_{p(b) k}^{*}\right\rangle-\left\langle f_{p(b) k}\right\rangle\left\langle f_{p(b) k}^{*}\right\rangle}, \\
& t_{k}^{b}=\sum_{i=1}^{2} \frac{\left|\nabla_{i} f_{b k}\right|^{2}}{2 m}, \quad t_{k}^{p}=\frac{\left|\nabla f_{p k}\right|^{2}}{2 m}, \\
& L_{p k}=\exp (i k r), \quad L_{b k}=\exp \left(i k r_{1}\right)+\exp \left(i k r_{2}\right) .
\end{aligned}
$$

Values $f_{k}$ are variational functions. Further calculations can be performed after the choice of the variational function, which will be used to compute the expressions included in the energy functionals for polaron or bipolaron. We choose the variational function $f_{k}$ in the form

$$
f_{k p(b)}=L_{k p(b)}^{*} .
$$

We also introduce the notation that will simplify the above rather cumbersome formula for the ground state

$$
\begin{aligned}
& P_{12}=\left\langle f_{p(b) k} L_{p(b) k}^{*}\right\rangle-\left\langle f_{p(b) k}\right\rangle\left\langle L_{p(b) k}^{*}\right\rangle, \\
& \widetilde{P}_{12}=\left\langle f_{p(b) k} f_{p(b) k}^{*}\right\rangle-\left\langle f_{p(b) k}\right\rangle\left\langle f_{p(b) k}^{*}\right\rangle .
\end{aligned}
$$

Further calculations can be made for the variation function of polaron or bipolaron selected in the given form. The above formulas are needed to calculate various contributions to the energy of bipolaron:

$$
\begin{aligned}
& \delta E_{b}=\delta T_{b}+\delta E_{\mathrm{int}}^{b}+\delta E_{P h}^{b}= \\
& =-\sum_{k} V_{k}^{2} \frac{\left|P_{12}\right|^{2}}{t_{p k}^{b}+\omega \widetilde{P}_{12}}=-\frac{4 \alpha}{\pi} \int_{0}^{\infty} \frac{U_{12}(k)^{2}}{k^{2}+U_{12}(k)} d k, \\
& U_{12}(k)=1-2 J_{0}(k)^{2}+J_{12}(k), J_{0}(k)= \\
& =\int\left|\Psi_{12}\right|^{2} \exp \left(i \mathbf{k r}_{1}\right) d \tau_{12}, \\
& J_{12}(k)=\int\left|\Psi_{12}\right|^{2} \exp \left(i \mathbf{k r}_{12}\right) d \tau_{12},
\end{aligned}
$$

where $t_{k}^{b}=\frac{k^{2}}{m \omega}, T_{b}=-\frac{\hbar^{2}}{2 m} \int\left(\nabla_{1}^{2}+\nabla_{2}^{2}\right)\left|\Psi_{12}\right|^{2} d \tau_{12}$,

$$
E_{\text {int }}^{b}=-\frac{4 \alpha}{\pi} \int_{0}^{\infty} J_{0}(k)^{2} d k
$$$$
E_{P h}^{b}=\frac{2 \alpha}{\pi} \int_{0}^{\infty} J_{0}(k)^{2} d k,
$$$$
E_{\mathrm{int}}^{b}=-\frac{4 \alpha}{\pi} \int_{0}^{\infty} J_{0}(k)^{2} d k,
$$

$\delta T_{b}=\sum_{k} V_{k}^{2} t_{k}^{b} \frac{P_{12}(k)^{2}}{\left[t_{k}^{b}+\omega \widetilde{P}_{12}(k)\right]^{2}}=$

$=\sum_{k} \frac{V_{k}^{2} k^{2}}{m \omega^{2}} \frac{P_{12}(k)^{2}}{\left[\frac{k^{2}}{m \omega}+\widetilde{P}_{12}(k)\right]^{2}}=$

$$
=\frac{4 \alpha}{\pi} \int_{0}^{\infty} \frac{U_{12}(k)^{2}}{\left[k^{2}+U_{12}(k)\right]^{2}} k^{2} d k,
$$

Table 1. Different contributions to the total energy of the bipolaron ground state. $\Psi_{12} \equiv \Psi_{12}\left(r_{1}, r_{2}\right)=$ $N\left(1+\gamma r_{12}^{2}\right) \exp \left(-\mu\left(r_{1}^{2}+r_{2}^{2}\right)\right)$. Energy is given in effective Hartree $H a^{*}=\hbar^{2} / a_{0}{ }^{2} m^{*}, a_{0}=\hbar^{2} \widetilde{\varepsilon} / m^{*} e^{2}, \widetilde{\varepsilon}^{-1}=\varepsilon_{\infty}^{-1}-\varepsilon_{0}^{-1}$ (Feynman energy unit $\hbar \omega=H a^{*} / 2 \alpha^{2}$ ).

\begin{tabular}{|l|c|c|c|c|c|c|}
\hline$\alpha$ & 7 & 8 & 9 & 10 & 15 & 20 \\
\hline$-E^{0}$ & 0.159593 & 0.152337 & 0.147437 & 0.143963 & 0.135835 & 0.133019 \\
\hline$-F$ & 0.128335 & 0.128851 & 0.129087 & 0.129212 & 0.129382 & 0.129409 \\
\hline$E_{\text {kin }}$ & 0.128335 & 0.128851 & 0.129080 & 0.129212 & 0.129383 & 0.129409 \\
\hline$E_{P h}^{\mathrm{C}} / 2$ & 0.112706 & 0.117106 & 0.119910 & 0.121836 & 0.129441 & 0.127603 \\
\hline$-E_{\text {el }} / 3$ & 0.128335 & 0.128851 & 0.129085 & 0.129212 & 0.129383 & 0.129409 \\
\hline$-E_{\text {int }} / 4$ & 0.128335 & 0.128851 & 0.129084 & 0.129212 & 0.129383 & 0.129409 \\
\hline
\end{tabular}

\begin{tabular}{|c|c|c|c|c|}
\hline$\alpha$ & 50 & 100 & 200 & $\alpha \rightarrow \infty$ \\
\hline$-E^{0}$ & 0.129994 & 0.129564 & 0.129456 & 0.129420 \\
\hline$-F$ & 0.129420 & 0.129420 & 0.129420 & 0.129420 \\
\hline$E_{\text {kin }}$ & 0.129420 & 0.129420 & 0.129420 & 0.129420 \\
\hline$E_{P h}^{\mathrm{C}} / 2$ & 0.129132 & 0.129270 & 0.129402 & 0.129420 \\
\hline$-E_{e l} / 3$ & 0.129420 & 0.129420 & 0.129420 & 0.129420 \\
\hline$-E_{\text {int }} / 4$ & 0.129420 & 0.129420 & 0.129420 & 0.129420 \\
\hline
\end{tabular}

(C) 2014, V. Lashkaryov Institute of Semiconductor Physics, National Academy of Sciences of Ukraine 
Table 2. The ground state energy of the bipolaron and values of variational parameters bipolaron wave function for various parameters of the Fröhlich coupling constant. Energy unit is $\hbar \omega$, the unit of length is $L_{0}=\sqrt{\hbar / 2 m^{*} \omega}, \hbar=1, \omega=1,2 m^{*}=1$.

\begin{tabular}{|l|l|l|l|}
\hline$\alpha$ & $\mu$ & $\gamma$ & $-E^{0}$ \\
\hline 7 & 1.96536431 & 1.59748281 & 15.640148 \\
\hline 8 & 2.7862314 & 2.2722311 & 19.499196 \\
\hline 9 & 3.70608514 & 3.02842228 & 23.884708 \\
\hline 10 & 4.72947124 & 3.86989438 & 28.792678 \\
\hline 15 & 11.42912057 & 9.37860362 & 61.125685 \\
\hline 20 & 20.79242068 & 17.07742448 & 106.415533 \\
\hline 50 & 133.10153731 & 109.42434937 & 649.973127 \\
\hline 100 & 534.18890865 & 439.21965692 & 2591.27627 \\
\hline 200 & 2138.53636 & 1758.40582 & 10356.4928 \\
\hline
\end{tabular}

$$
\begin{aligned}
& \delta E_{\mathrm{int}}^{b}=-\sum_{k} \frac{2 V_{k}^{2}}{\omega} \frac{P_{12}(k)^{2}}{\frac{k^{2}}{m \omega}+\widetilde{P}_{12}(k)}=-\frac{8 \alpha}{\pi} \int_{0}^{\infty} \frac{U_{12}(k)^{2}}{k^{2}+U_{12}(k)} d k, \\
& \delta E_{P h}^{b}=\sum_{k} \frac{V_{k}^{2}}{\omega} \frac{\widetilde{P}_{12}(k) P_{12}(k)^{2}}{\left[\frac{k^{2}}{m \omega}+\widetilde{P}_{12}(k)\right]^{2}}=\frac{4 \alpha}{\pi} \int_{0}^{\infty} \frac{U_{12}(k)^{3}}{\left[k^{2}+U_{12}(k)\right]^{2}} d k .
\end{aligned}
$$

We perform numerical illustration of the virial theorem fulfillment for Pekar bipolaron for the one of the simplest wave function $\Psi_{12} \equiv \Psi_{12}\left(r_{1}, r_{2}\right)=$ $N\left(1+\gamma r_{12}^{2}\right) \exp \left(-\mu\left(r_{1}^{2}+r_{2}^{2}\right)\right)$, admitting to obtain a sufficiently accurate value of the bipolaron energy for intermediate electron-phonon coupling (Tables 1,2).

\section{Conclusion}

Numerical check performed by us demonstrates that the virial relations (15) are fulfilled for bipolaron considered using the Buimistrov-Pekar method for an arbitrary value of the electron-phonon interaction. As in the case of strong coupling limit, the virial theorem holds true for both the one-center bipolaron configuration and the twocenter one corresponding to a shallow subsidiary minimum that disappears when the electron wave function is chosen with regard for electron correlations associated with the direct dependence of the wave function on the distance between electrons. Therefore, V.K. Mukhomorov's statement [16] that the virial theorem does not hold true for one-center polaron (as distinct from the case of two-center polaron), and the statement that for the virial theorem to hold true the variational principle should be supplemented by a condition that would limit from below the functional under variation by the virial relation are erroneous (see also comments [29]). At the points corresponding to minimum values of the functional, the virial theorem holds true automatically. According to the variational principle, preference should be given to a lower energy value, since higher values can disappear when a more versatile wave function is chosen (see for example p. 49 in ref. [8]).

The last work by V. L.Vinetskii performed with his colleagues was made for the one-center bipolaron model [30]. Research issues related to the spatial configuration of bipolaron in low-dimensional systems and anisotropic crystals deserves the separate consideration because such systems have a preferred direction.

The author wishes to express her appreciation of valuable advice and encouragement afforded by Professor V.D. Lakhno and Professor E.A. Pashitskii.

\section{References}

1. G. Verbist, F.M. Peeters, J.T. Devreese, Large bipolarons in two and three dimensions // Phys. Rev. B, 43(4), p. 2712-2720 (1991).

2. J. Adamowski, S. Bednarek, Stability of large bipolarons // J. Phys.: Condens. Matter, 4(11), p. 2845-2855 (1992).

3. J. Adamowski, Formation of Fröhlich bipolarons // Phys. Rev. B, 39(6), p. 3649-3652 (1989).

4. N.I. Kashirina, V.D. Lakhno, V.V. Sychyov, Correlation effects and Pekar bipolaron (arbitrary electron-phonon interaction) // Physica status solidi (b), 239(1), p. 174-184 (2003).

5. R.A. Ogg, Bose-Einstein condensation of trapped electron pairs. Phase separation and superconductivity of metal-ammonia solutions // Phys. Rev. 69 , p. 243-243 (1946).

6. R.A. Ogg, Physical interaction of electrons with liquid dielectric media. The properties of metalammonia solutions // Phys. Rev. 69, p. 668-668 (1946).

7. R.A. Ogg, Superconductivity in solid metalammonia solutions // Phys. Rev. 70(1), p. 93-93 (1946).

8. S.I. Pekar, Research in Electron Theory of Crystals. GITTL Publ., Moscow-Leningrad, 1951 (in Russian). [S.I. Pekar, Research in Electron 
Theory of Crystals. United States Atomic Energy Commision, Division of Technical Information, Washington, 1963].

9. O.F. Tomasevich, $F^{\prime}$-centers in alkali-halide crystals // Zhurnal Eksp. Teor. Fiziki, 21(11), p. 1223-1227 (1951), in Russian.

10. A.S. Davydov, On the theory of the magnetic properties of metal-ammonia solutions // Nauchnye zapiski Kievskogo gos. universiteta. XI(IV); Trudy fizich. fakult. Kiev. gos. univ. 5 (1), p. 26-31 (1952), in Russian.

11. V.L. Vinetskii, M.S. Giterman, To the theory of added charges interaction in polar crystals // Zhurnal Eksp. Teor. Fiziki, 33(3), p. 730-734 (1957), in Russian.

12. V.L. Vinetskii, About bipolaron states of charge carriers in ionic crystals // Zhurnal Eksp. Teor. Fiziki, 40(5), p. 1459-1468 (1961), in Russian.

13. M.F. Deigen, Thermal dissociation and the ground state energy of the double color center in ionic crystals // Zhurnal Eksp. Teor. Fiziki, 21(9), p. 992-1000 (1951), in Russian.

14. T. Masumi, A supplemental improvement of Feynman-polaron model and the possibility of bipolaron formation // Suppl. Progr. Teor. Phys. 57, p. 22-34 (1975).

15. S.G. Suprun and B.Ya. Moyzhes, About the role of electron correlation in the formation of Pekar bipolaron // Fizika Tverd. Tela, 24(5), p. 1571-1573 (1982), in Russian.

16. V.K. Mukhomorov, Stability bipolaron formations, electron-electron correlations, the variational principle and the virial theorem // Fizika Tverd. Tela, 48(5), p. 814-820 (2006), in Russian.

17. N.I. Kashirina, V.D. Lakhno, The spatial configuration of the bipolaron and virial theorem // Fizika Tverd. Tela, 50(1), p. 11-16 (2008), in Russian.

18. J.C. Slater, Quantum Theory of Molecules and Solids, Vol. 1: Electronic Structure of Molecules. McGraw-Hill, New York, 1963.

19. D.M. Larsen, Giant binding of $\mathrm{D}^{-}$centers in polar crystals // Phys. Rev. B, 23 (2), p. 628-631 (1981).
20. M.H. Cohen, E.N. Economou, C.M. Soukoulis, Small-bipolaron formation // Phys. Rev. B, 29, p. 4496-4499 (1984).

21. P.Zh. Bajmatov, D.Ch. Huzhakulov, H.T. Sharipov, Bipolaron in the intermediate coupling method // Fizika Tverd. Tela, 39(2), p. 284-285 (1997), in Russian.

22. N.I. Kashirina, V.D. Lakhno, V.V. Sychyov, Electron correlations and spatial configuration of bipolaron // Physica status solidi (b), 234(2), p. 563-570 (2002).

23. N.I. Kashirina, V.D. Lakhno, V.V. Sychyov, Polaron effects and electron correlations in twoelectron systems: Arbitrary value of electronphonon interaction // Phys. Rev. B, 71, 134301-1134301-13 (2005).

24. V.M. Buimistrov, S.I. Pekar, Quantum states of particles interacting with a harmonically oscillating continuum for an arbitrary coupling strength. 1 . There is no translational symmetry the case // Zhurnal Eksp. Teor. Fiziki, 32, p. 1193-1199 (1957), in Russian.

25. L.F. Lemmens, J.T. Devresse, The 1:2:3:4 theorem and the ground state of free polarons // Solid State Communs. 12(10), p. 1067-1069, (1973).

26. P. Gombash, Problem of Many Particles in Quantum Mechanics. Inostr. Lit. Publ., Moscow (1952), in Russian.

27. J. Röseler, A new variational ansatz in the polaron theory // Physica status solidi (b), 25 (1), p. 311316 (1968).

28. R.P. Feinman, Forces in molecules // Phys. Rev. 56, p. 340-343 (1939).

29. N.I. Kashirina, V.D. Lakhno, Comments on the Series of Works by V.K. Mukhomorov on the Theory of Continual Polaron and Two-Center Bipolaron (Axisymmetrical Quasi-Molecular Dimer) // Mat. Biol. and Bioinf. 2(2), p. 10-28 (2007).

30. V.L. Vinetskii, O. Meredov and V.Ja. Janchuk, Quantum chemistry of bipolaron in the isotropic continuum medium // Teor. Eksp. Him. 25(6), p. 641-647 (1989), in Russian. 